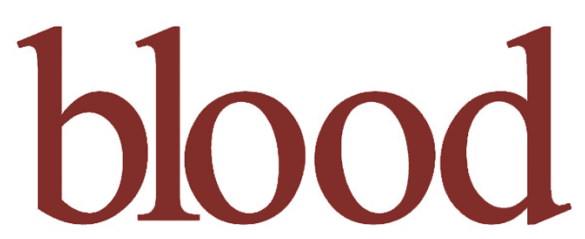

Prepublished online Apr 6, 2009;

doi:10.1182/blood-2008-12-196543

\title{
Prevalence, determinants, and outcomes of nonadherence to imatinib therapy in patients with chronic myeloid leukemia: the ADAGIO study
}

Lucien Noens, Marie-Anne van Lierde, Robrecht De Bock, Gregor Verhoef, Pierre Zachee, Zwi

Berneman, Philippe Martiat, Philippe Mineur, Koen Van Eygen, Karen MacDonald, Sabina De Geest, Tara Albrecht and Ivo Abraham

Information about reproducing this article in parts or in its entirety may be found online at: http://bloodjournal.hematologylibrary.org/misc/rights.dt|\#repub_requests

Information about ordering reprints may be found online at:

http://bloodjournal.hematologylibrary.org/misc/rights.dt|\#reprints

Information about subscriptions and ASH membership may be found online at:

http://bloodjournal.hematologylibrary.org/subscriptions/index.dtI

Blood (print ISSN 0006-4971, online ISSN 1528-0020), is published semimonthly by the American Society of Hematology, 1900 M St, NW, Suite 200, Washington DC 20036.

Copyright 2007 by The American Society of Hematology; all rights reserved.

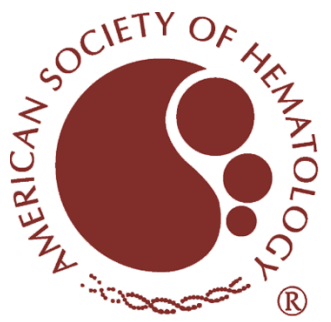


Left Running Head: $\quad$ NOENS et al.

Right Running Head: $\quad$ NONADHERENCE TO IMATINIB IN CML PATIENTS

Scientific Section Designation: CLINICAL TRIALS AND OBSERVATIONS

Prevalence, determinants, and outcomes of nonadherence to imatinib therapy in patients with chronic myeloid leukemia: the ADAGIO study

Lucien Noens, ${ }^{1}$ Marie-Anne van Lierde, ${ }^{2}$ Robrecht De Bock, ${ }^{3}$ Gregor Verhoef,4 Pierre Zachée, ${ }^{5}$ Zwi Berneman, ${ }^{6}$ Philippe Martiat, ${ }^{7}$ Philippe Mineur, ${ }^{8}$ Koen Van Eygen, ${ }^{9}$ Karen MacDonald, ${ }^{10}$ Sabina De Geest, ${ }^{11}$ Tara Albrecht,,10,12 Ivo Abraham ${ }^{10,13}$

1UZ Gent, Gent, Belgium; ${ }^{2}$ Novartis Pharma, Vilvoorde, Belgium; ${ }^{3 Z N A}$ Middelheim, Antwerpen, Belgium; ${ }^{4}$ UZ Gasthuisberg, Leuven, Belgium; ${ }^{5 Z N A}$ Stuivenberg, Antwerpen, Belgium; ${ }^{6} \mathrm{UZA}$, Antwerpen, Belgium; ${ }^{7}$ Institut Jules Bordet, Bruxelles, Belgium; ${ }^{8}$ Hôpital St. Joseph, Gilly, Belgium; ${ }^{9}$ Z Groeninge, Kortrijk, Belgium; ${ }^{10}$ Matrix45; Earlysville, VA, USA;

${ }^{11}$ University of Basel, Institute of Nursing Science, Basel, Switzerland; ${ }^{12}$ University of Virginia, School of Nursing, Charlottesville, VA, USA; ${ }^{13}$ University of Arizona, College of Nursing, and Center for Health Outcomes and PharmacoEconomic Research, College of Pharmacy, Tucson, AZ, USA

Presented in various parts in abstract form at the $14^{\text {th }}$ European Cancer Conference, Barcelona, Spain, September 2007; the 49th annual meeting of the American Society of Hematology, Atlanta, GA, USA, December 2007; the $13^{\text {th }}$ congress of the European Haematological Association, Copenhagen, Denmark, June 2008; and the 50 th annual meeting of the American Society of Hematology, San Francisco, CA, USA, December 2008. 


\begin{abstract}
Imatinib mesylate (imatinib) has been shown to be highly efficacious in the treatment of chronic myeloid leukemia (CML). Continuous and adequate dosing is essential for optimal outcomes and with imatinib treatment possibly being life-long, patient adherence is critical. The ADAGI0 study aimed to assess prospectively over a 90-day period the prevalence of imatinib nonadherence in CML patients; to develop a multivariate canonical correlation model of how various determinants may be associated with various measures of nonadherence; and to examine whether treatment response is associated with adherence levels. A total of 202 patients were recruited from 34 centers in Belgium, of whom 169 were evaluable. One-third of patients were considered to be nonadherent. Only $14.2 \%$ of patients were perfectly adherent with $100 \%$ of prescribed imatinib taken. On average, patients with suboptimal response had significantly higher mean percentages of imatinib not taken $(23.2 \%, \mathrm{SD}=23.8)$ than did those with optimal response $(7.3 \%, \mathrm{SD}=19.3$, $\mathrm{P}=0.005$; percentages calculated as proportions $\mathrm{x} 100$ ). Nonadherence is more prevalent than patients, physicians, and family members believe it is, and therefore should be assessed routinely. It is associated with poorer response to imatinib. Several determinants may serve as alert signals, many of which are clinically modifiable.
\end{abstract}


Introduction

Imatinib mesylate (imatinib) is a major advance in the pharmacological treatment of chronic myeloid leukemia (CML) with regard to efficacy and safety ${ }^{1}$. Imatinib blocks the ATP-binding site of the BCR-ABL tyrosine kinase with high selectivity² and has been found effective in the chronic ${ }^{3}$ and accelerated phases of $\mathrm{CML}^{4,5}$, as well as in blast crisis ${ }^{6}$. Longterm follow-up studies have shown that imatinib's therapeutic benefits, initially documented for up to 18 months $^{7}$, may extend to seven years ${ }^{8}$ in continuously treated chronic phase CML patients. Imatinib 400mg daily has been recommended as first-line treatment for patients newly diagnosed in chronic phase of the disease ${ }^{9}$, in part also because of the impact on quality of life ${ }^{10,11,12}$ and favorable cost-efficacy ${ }^{13}$ and costeffectiveness $12,14,15$

Continuous and adequate imatinib dosing is essential to achieve therapeutic outcomes. ${ }^{16}$ Hence, patient adherence, defined as the extent to which a person's behavior corresponds with the agreed recommendations of a healthcare provider ${ }^{17}$, is critical. Though often trivialized as a patient problem, adherence behavior is influenced also by the clinician and the healthcare system, the disease and its treatment, and economic and social factors. ${ }^{17}$

Retrospective analyses of claims data in the US provide some preliminary evidence about adherence to imatinib. An analysis of 374 patients with CML and 91 patients with gastrointestinal stromal tumors (GIST) with at least 12 months of treatment found mean and median persistence rates across all patients of $69.4 \%$ and $79.7 \%$, respectively (no disease-specific rates_were reported). ${ }^{18}$ Another analysis reviewed 267 CML patients in their first year of treatment with imatinib. ${ }^{19}$ The mean medication possession ratio (MPR, defined as total days supply of imatinib divided by 365 ) in the first year was $77.7 \%$. MPR was lower among female patients, patients taking relatively more concomitant medications, 
those with high cancer complexity, and those started on imatinib doses of $600 \mathrm{mg} /$ day or higher. Female patients and those with high cancer complexity were about twice as likely to interrupt treatment. In addition, $30.7 \%$ failed to refill imatinib within 30 days; however all patients with treatment interruptions resumed imatinib treatment within the 12-month period. In both studies ${ }^{18,19}$ lower imatinib adherence was associated with higher medical expenditures.

A case-control study evaluated the adherence (measured by pill count converted to mg taken / mg prescribed x 100) of 21 evaluable CML patients in their first year of imatinib treatment and its association with cytogenetic response. ${ }^{20}$ Patients were matched for sex, age, and hematologic response with controls from an existing database. The mean adherence rate for the cases during the 12 -month period was $96.1 \% \pm 9 \% .89 .9 \% \pm 20 \%$ of cases showed major cytogenetic response (defined as $<35 \% \mathrm{Ph}$-positive metaphases) compared to $60 \% \pm 25 \%$ for controls (for whom no adherence data were available). Rates for complete cytogenetic response were not reported.

To our knowledge, no prospective studies of patient adherence with imatinib treatment have been published in full. The ADAGIO study (Adherence Assessment with Glivec $($ : Indicators and Outcomes) aimed (1) to examine prospectively over a 90-day period, in the "real practice" setting, the prevalence of imatinib nonadherence in CML patients in Belgium on imatinib treatment for at least 30 days; (2) to develop a multivariate canonical correlation model of how various determinants may be associated with various measures of nonadherence; (3) and to examine whether treatment response is associated with adherence levels. 
Patients and methods

Design and sample

This study was designed as a prospective, observational, multi-center, noninterventional study with two time points: baseline (enrollment visit) and follow-up approximately 90 days later. Eligible were male and female patients, at least 14 years old, diagnosed with CML, and on imatinib treatment within the approved label for at least 30 days (to enable assessment of adherence prior to the observational period). Excluded were patients with known sensitivity to imatinib, patients not treated within the approved label, and patients with a severe medical condition that in the view of the investigator prohibited participation in the study. Patients were allowed to take other medications and to continue or discontinue these medications at any point in time during the study period as this was an observational study not intended to interfere with physicians' clinical practice. A total of 202 patients were recruited by 51 physician-investigators at 34 centers in Belgium.

The study protocol was approved by the medical ethics committee of the University Hospital Gent (UZ Gent, Gent, Belgium) as well as the medical ethics committee at each participating center. All patients gave written informed consent in accordance with the Helsinki protocol.

Variables and measurement methods

Table 1 summarizes the variables included in this study. The complete data model and English translations of the case record forms are available from the corresponding author. Major variables and measurement methods are specified below. 
Disease parameters. Hematologic response was defined as complete response (leukocyte count $<10 \times 10^{9} / \mathrm{L}$, platelet count $<450 \times 10^{9} / \mathrm{L},<5 \%$ myelocytes plus metamyelocytes, no blasts or promyelocytes, no extramedullary involvement, and no CML accelerated phase or blast crisis), no evidence of leukemia, or return to chronic phase. Cytogenetic response in bone marrow cells was defined as complete $(0 \% \mathrm{Ph}$-positive metaphases), partial (1-35\% Ph-positive metaphases), or major (complete plus partial responses). Molecular response was expressed as $\geq 3$ and $\geq 2$ log reductions in the BCR$\mathrm{ABL} / \mathrm{BCR}$ ratio per local laboratory without standardization. In accordance with the European LeukemiaNet criteria ${ }^{9}$ suboptimal response was defined at 3 months as incomplete hematologic response; at 6 months as less than partial cytogenetic response; and at 18 months as less than major molecular response and, in case of loss of major molecular response, other limitations or other chromosomal abnormalities.

Adherence. The WHO definition of adherence, being the extent to which a person's behavior corresponds with the agreed recommendations of a healthcare provider ${ }^{17}$, was operationalized along the behavioral dimensions of taking and timing adherence, occurrence of drug holiday(s), and/or reduction in dose of medication. Given the absence of a gold standard of adherence measurement ${ }^{21,22}$, the varying benefits and limitations of available methods ${ }^{22}$, and keeping clinical utility in mind, several methods were used to assess adherence. This is congruent with the Osterborg and Blaschke ${ }^{23}$ recommendation to combine several assessment methods.

At both baseline and follow-up, physicians used the Basel Assessment of Adherence Scale with Immunosuppressive Medication ${ }^{24}$ adapted to imatinib. This scale (referred to here as BAAS) is a 4-question clinical interview guide: a positive answer to any questions constitutes nonadherence. Physicians also used the BAAS to assess patient adherence as perceived by a third person such as spouse or other family member (as available). 
Physicians, patients, and third persons rated patient adherence on a $10 \mathrm{~cm}$ visual analogue scale (VAS) converted to a 0-100 score. Adherence with scheduled appointments was measured as the ratio of appointments scheduled to appointments kept. At follow-up, a pill count was performed and expressed as the percentage of imatinib taken to imatinib prescribed.

Patient scales. At baseline, patients completed the Long-Term Medication Behavior Self-Efficacy scale (LMBSE).25,26 Self-efficacy refers to a person's level of confidence in performing a specific behavior. ${ }^{27}$ The self-efficacy score is calculated by summing scores on all items divided by the number of items. Self-efficacy scores thus range from 1 to 5 , with higher scores indicating higher levels of self-efficacy. At both baseline and follow-up, the Patient Assessment of Chronic Illness Care 28,29 (PACIC) scale was used to measure patients' perceptions of the degree of chronic care they received; and the SF-8 Health Survey ${ }^{30}$ (nonstandardized) to assess functional status and quality of life. Patients were also queried about their understanding of disease and treatment and their methods of informationseeking. Patients were asked to complete these scales at the time of the respective visit (Table 1). To protect the confidentiality of their responses, completed instruments were returned in a sealed envelope to center staff.

Physician-investigator experience and perceptions. At baseline physicianinvestigators were queried, among other things, about the number of active CML patients seen in the past year and the median time they spend with newly diagnosed patients and patients in follow-up. They were asked to rank-order various sources of information and modes of decision-making about managing CML, as well as various modes of patient interaction and support. Physicians estimated the percentages of patients adherent with imatinib treatment in the first month following diagnosis and after one year; and the 
percentage of imatinib-treated patients who do not achieve therapeutic effect because, and only because, of poor adherence. Physicians also rated the relevance of several potential determinants of adherence behavior: patient demographic, social, and economic factors; patient-physician relationship; treatment; disease; physician; and other patient variables (knowledge, attitudes, and feelings about disease and treatment; participation in treatment; multiple comorbidities; lifestyle; and mental health). Lastly, physicians rated the effectiveness, feasibility, cost, and clinical applicability of thirteen adherence-enhancing interventions.

Statistical analysis

Prevalence was estimated as the period prevalence rate for the time period indicated, using the evaluable sample of patients as the denominator.

Considering that patients were "nested" under physicians and centers, unconditional hierarchical linear modeling (HLM) with estimation using residual maximum likelihood (REML) was applied to examine the class effects of physician or center. The intraclass coefficient (ICC) quantifies the proportion of variability accounted for by the class being examined and was used to attribute the respective percentages of variance in continuous adherence measures to class (ICCx100) and patients ([1-ICC]x100).

To permit the use of a multivariate definition of nonadherence, canonical correlation analysis (CCA) was used to multivariately model the relationship of a vector composed of three complementary measures of nonadherence with a vector comprising patient- and physician-related determinants. CCA has been termed the multivariate analog of multiple linear regression and accommodates two or more criterion ("dependent") variables compared to the univariate limitation of one criterion variable in multiple linear regression. ${ }^{31}$ While one could construct a composite index of nonadherence as, for instance, 
the (weighted) sum of the three variables included here, a multivariate representation as a vector enables consideration of all covariations among variables through the covariance matrix. Thus it measures a total that is more than the sum of its parts. The combination of different measurement methods in one vector is congruent with Osterberg's and Blaschke's recommendation. ${ }^{23}$ A non-adherence vector was specified with the following elements: inverse patient VAS rating (self-perception), patient BAAS score (self-report), and inverse pill count. Inversions were done so that VAS rating and pill count were expressed isodirectionally with the BAAS. The statistical significance of canonical correlation coefficients derived was determined by Wilk's test that remaining correlations are zero. Also calculated for the vector of determinants were the canonical loadings, the percent of variance explained, and the redundancy statistic. The model was directional in that variability in the nonadherence vector was assumed to be a function of variability in the vector of determinants, hence the calculation and reporting of parameters for the latter vector.

For comparisons between two subgroups of patients classified on the basis of treatment response the $t$-test or its nonparametric analog (Mann-Whitney $U$ ) were used, with Bonferroni class corrections to manage multiplicity. Baseline to follow-up comparisons were done using the paired $t$-test or its nonparametric analog (Wilcoxon signed-rank test). Continuity corrections were applied in contingency table analyses of $2 \times 2$ tables. Associations between adherence and other variables were determined, depending on the levels of measurement of the variables involved, by means of the Pearson product moment correlation coefficient, the Spearman rho coefficient, or the point bi-serial coefficient.

For all statistical calculations, the significance level $\alpha$ was set at 0.05 . Using multiple power and precision calculations given the various objectives, required sample size estimates ranged from 126 to 162 evaluable patients to achieve power of 0.80 at $\alpha=0.05$. 
Results

Patient characteristics

A total of 169 patients contributed by 51 physician-investigators at 34 centers completed the study with evaluable data. Evaluable was defined as meeting inclusion criteria and having baseline and follow-up data (including the physician's evaluation, patient interview and patient questionnaire), pill counts at end of study, and data on practice characteristics from the corresponding physician questionnaire. All patients were required to have at least one of the outcome variables of physician-rated adherence or patient-rated adherence. Reasons for being considered non-evaluable included lack of baseline patient questionnaire (2.0\%), follow-up patient questionnaire $(6.4 \%)$, follow-up patient interview $(0.5 \%)$, pill count $(8.4 \%)$, or physician questionnaire $(2.0 \%)$ as well as absence of both physician-rated and patient-rated adherence measure (8.4\%). The evaluable sample size enabled estimation of parameters with adequate precision and permitted statistical analysis with power of at least 0.80 at $\alpha=0.05$.

The mean age was $57.2 \pm 14.5$ years (Table 2 ). The majority of patients were male (55.0\%) and educated at lower or higher secondary school level (64.6\%). Virtually all patients were white (96.4\%). Most lived with family (78.7\%), however 19.5\% resided alone at home and a few $(1.8 \%)$ in supportive care facilities.

The median and mean times from diagnosis of CML were 41.9 months and 48.8 \pm 41.4 months, respectively (from 1.6 to 347 months; $95 \%$ CI 41.4 to 56.2 ); $45.1 \%$ were symptomatic at the time of diagnosis. At baseline, almost all patients had primary CML disease (93.3\%) and were in chronic phase (98.2\%). Hematologic, cytogenetic, and molecular responses were documented as available. There were 147 patients (87.0\%) with a complete hematologic response, 7 (4.1\%) had no evidence of leukemia (complete 
hematological response with absolute neutrophil count $>1000 / \mathrm{mm}^{3}$ and platelet count $>20,000 \mathrm{~mm}^{3}$ ), and none had returned to chronic phase (note, $8.9 \%$ had no documented hematologic response). Further, 129 patients (76.3\%) had a cytogenetic response at baseline, including 110 (65.1\%) with complete response. There were $47.9 \%$ of patients who had $\mathrm{a} \geq 3 \log$ reduction in $\mathrm{BCR}-\mathrm{ABL} / \mathrm{BCR}$ ratio and $13.6 \%$ showing $\geq 2 \log$ reduction (another $38.5 \%$ had no documented molecular response). These parameter proportions did not change in a statistically significant manner from baseline to follow-up (Table 3).

Most common prior CML treatments included interferon $(64.2 \%$ of patients; of these, $28.0 \%$ experienced treatment failure and $13.0 \%$ relapsed, i.e., loss of response, following initial treatment success) and hydroxycarbamide (77.1\%; treatment failure rate was $40.3 \%$, relapse rate $27.4 \%$ ); $54.4 \%$ of patients had received both prior to imatinib treatment. For $14.8 \%$ of patients imatinib was the first line of treatment. The median and mean duration of exposure to imatinib were 35.2 and $32.9 \pm 19.8$ months respectively (from 1.0 to 72.8 months; $95 \%$ CI 29.8 to 36.1 ). There were minimal, statistically not significant differences in the mean imatinib dosages at initiation of treatment, start of study, and follow-up. With the exception of $9.5 \%$ of patients taking paracetamol, no patients were prescribed any other agents known to interact with imatinib.

Most frequent (in $\geq 15 \%$ of patients) past or current comorbidities were gastrointestinal (28.4\%), skeletal (26.6\%), cardiac (22.5\%), and endocrine/metabolic disease (16.0\%). About (40.8\%) of patients had a history of smoking (incl. 10.9\% active) and $32.7 \%$ a family history of cancer.

During the 90-day study period, imatinib dose was changed in seven (4.1\%) and discontinued in three (1.8\%) patients, mainly (70\%) because of intolerance to the medication (Table 2).

Patient-reported outcomes 
At baseline, patients' SF-8 scores for functional status and quality of life ranged from 14 to 42 on a $8-42$ nonstandardized scale $(M=31.5, S D=6.5)$. Patient ratings of the degree of chronic care (PACIC) received ranged from 1 to 5 on a $1-5$ scale $(M=2.8, S D=0.9)$. Selfefficacy of long-term medication behavior (LMBSE) ratings were between 1.8 and 5 on a 1-5 scale $(M=4.7, S D=0.5)$. Patient's knowledge of disease and treatment was observed across the full range of the $0-100$ scale $(M=71.9, S D=20.3)$ (Tables 2 and 3). There were no statistically significant changes from baseline to follow-up for functional status/quality of life and knowledge of disease and treatment. Patients' perceptions of chronic care decreased from baseline to follow-up $(p=0.01)$.

Physician variables

Physician-investigators ranged in age from 29 to 65 years $(M=44.5 \pm 8.0)$ and had been practicing medicine between 4 and 35 years $(M=17.7 \pm 8.1)$. Most were male $(59.2 \%)$; $74.0 \%$ were hematologists versus $26.0 \%$ oncologists; working in university (46.9\%), university-affiliated (18.4\%), or non-university hospitals (34.7\%). They had seen between 1 and 50 active CML patients in the 12 months preceding the start of the study $(M=10.7 \pm 8.8) ;$ spending between 30 and 100 minutes on the first visit with a newly diagnosed CML patient $(M=43.9= \pm 15.3)$ and between 10 and 30 minutes on treatment follow-up visits $(M=20.2 \pm 5.8)$.

When asked to rate various strategies to enhance patients' involvement in the management of their disease, the modal ratings were, in descending order, encouraging patients to phone with questions (modal rating of 4 out of 5 by $51.0 \%$ of physicians), explaining the importance of treatment adherence (mode=3; 45.1\%), talking to the patient about medication dosing and side effects (mode $=2 ; 45.1 \%$ ) and talking to the patient about 
the disease process (mode $=1 ; 52.9 \%$ ). Hand-outs and related patient materials received modal ratings of 0 , whether self-developed materials $(80.4 \%)$, materials designed by pharmaceutical companies $(60.8 \%)$, or received at a continuing medical education event $(54.9 \%)$.

Physicians believed that on average $92.8 \pm 13.1 \%$ (range $=20-100$ ) of patients were imatinib adherent in the first month after diagnosis and that $87.4 \pm 9.4 \%$ (range $=50-100$ ) were so after one year of treatment. They also reported that on average $8.0 \pm 6.4 \%$ (range 0 30) of patients do not achieve therapeutic effect because, and only because, of poor treatment adherence.

Further, physicians were asked to rate the various $\mathrm{WHO}^{17}$ categories of factors that impact on adherence as "not", "somewhat", or "strongly contributing" to nonadherence to imatinib therapy. We combined the latter two options to create a binary score of not contributing vs. contributing to nonadherence. Therapy-related factors were identified by the most physicians as determinants of non-adherence ( $96.1 \%$ of respondents), followed by patient demographic, social and economic factors (92.1\%), the patient-physician relationship (92.1\%), disease-related factors (84.3\%), physician-related factors $(70.6 \%)$, and other patient-related variables (70.5\%). Within the patient-physician relationship, the communication and interpersonal style of the physician as well as the continuity of care by the physician were identified by most respondents as a contributing variable (both $96.1 \%$ ), followed by time the physician spends with the patient ( $91.2 \%)$, physician empathy and assistance (89.2\%), and patient involvement in planning (88.3\%). As to physician-related variables that contribute to patient adherence, most respondents endorsed practicing in accordance with (92.2\%) and knowledge of practice guidelines (91.2\%), and to a lesser extent years of clinical experience in general (66.7\%) and in treating patients with chronic disease $(72.5 \%)$, or the number of patients with chronic disease seen regularly in practice $(66.7 \%)$ 
Physicians rated the effectiveness, feasibility, cost (all on a 0-3 scale) and clinical applicability (0-5 scale) of thirteen adherence-enhancing interventions (Table 4). 0n average, the highest (mean $\geq 2$ ) effectiveness, feasibility, and applicability ratings were given to improved patient-physician communication, patient education, simplifying the medication regimen, regular physician contact, spouse/family involvement, and monitoring of patient adherence by the physician; most of which were rated as low to medium cost.

Patient adherence with imatinib therapy

In general, VAS ratings of patient adherence by physicians, patients, and third persons (if available) were very high (94.9 to 97.1 on $0-100$ scale) at both baseline and follow-up. Differences within source over time were statistically not significant, and neither were differences between sources within time point.

Per the BAAS, $36.1 \%$ of patients at baseline and $32.7 \%$ at follow-up reported to have exhibited at least one of the four queried behaviors in the 4 weeks prior $(\mathrm{P}=\mathrm{ns})$. The most common behaviors were occasionally not taking a dose $(16.1 \%$ at baseline, $13.3 \%$ at followup) and taking a dose with a delay of more than two hours (22.2\% and $25.3 \%$, respectively).

For those patients with scheduled appointments, $89.4 \%$ had kept those appointments in the 30 days prior to baseline and $86.6 \%$ during the 90 -day study period. At baseline, mean percentage of appointments kept was $90.9 \% \pm 28.6$; at follow-up this was $92.1 \% \pm 20.1(\mathrm{P}=\mathrm{ns})$.

Pill counts were used to calculate the percent of total prescribed dose taken to prescribed during the 90 -day period. Scores ranged from $29 \%$ to $202 \%$ of prescribed dose $(M=90.9 \pm 20.1)$. 
Association of adherence with length of illness, duration of imatinib treatment, and safety and tolerance

We examined the associations of time since CML diagnosis, time on imatinib treatment, number of adverse events (general and suspected to be related to imatinib), and number of patient-reported symptoms and their bothersomeness with adherence at both enrollment (physician, patient, and third person VAS; BAAS) and at follow-up (physician, patient, and third person VAS; BAAS; pill count). There were no significant associations between adherence behavior and length of illness or duration of treatment at either enrollment or follow-up. With the exception of a weak correlation $\left(\mathrm{r}_{\mathrm{bs}}=-0.240, \mathrm{P}=0.007\right)$ between bothersomeness of symptoms and adherence behavior per the BAAS, all other associations of safety and tolerance variables with adherence (VAS and BAAS) were statistically not significant at enrollment. At follow-up, there were no statistically significant associations of general adverse events, imatinib-specific adverse events, number of patient-reported symptoms, and bothersomeness of these symptoms with pill count adherence. Weak correlations were observed between the number of adverse events and patient $(\mathrm{r}=0.166, \mathrm{P}=0.032)$ and third person $(\mathrm{r}=0.237, \mathrm{P}=0.045)$ perceptions of adherence (per VAS scales), and adherence behavior ( $\left.\mathrm{r}_{\mathrm{bs}}=-0.241, \mathrm{P}=0.002\right)$; and between the number of symptoms reported by patients and their perceptions of $(\mathrm{r}=0.208, \mathrm{P}=0.007)$ and actual adherence behavior $\left(\mathrm{r}_{\mathrm{bs}}=-0,211, \mathrm{P}=0.006\right)$.

Multivariate analyses

Though above we report adherence results for all measures, only those with data for at least 160 patients were included in multivariate analyses. 
Attribution of variance in adherence behavior. The ICCs for physician and center for patient VAS adherence ratings were 0.346 and 0.362 , respectively. For physician VAS adherence rating, the ICCs for physician and center were 0.069 and 0.120 . Physician ICC for pill count was 0.091; the center ICC for this adherence parameter was $<0.01$. Physician and center ICCs for the BAAS were 0.072 and 0.122 .

Canonical correlation modeling. A model including 12 patient-related and 6 physician-related determinants with either a negative or positive influence on nonadherence was fitted (Table 6). The canonical correlation was 0.509 (Wilk's=0.484, $\mathrm{P}=0.036)$. The model had minimal redundancy (0.015). Patient-related determinants associated with higher nonadherence were, in descending order of canonical loading: higher age (0.649), longer time since CML diagnosis (0.272), living alone (0.246), male gender (0.194), longer time on imatinib (0.193), imatinib dose $\geq 600 \mathrm{mg} /$ day (0.193), higher degrees of chronic care received (0.125), and higher self-reported functional status and quality of life (0.117). Physician-related determinants of higher nonadherence included median duration of treatment follow-up visits ( 0.237 ; presumably a proxy of vigilance) and years of professional experience $(0.135)$.

On the other hand, patient-related variables associated with better adherence (i.e., lower nonadherence) were knowledge of disease and treatment $(-0.314)$, more medications to be taken daily $(-0.184)$, secondary school or higher education $(-0.140)$, and self-efficacy in long-term medication behavior $(-0.062)$. Physician-related variables associated with a reduction in nonadherence included the number of active CML patients seen in the past year $(-0.363)$ and the median duration of the first visit with a newly diagnosed CML patient (-0.119); and to a lesser extent, practicing in a university or teaching hospital $(-0.003)$ and holding specialization in hematology $(-0.002)$. 
Nonadherence and treatment response

The nonadherence measure of pill count (expressed as percent not taken of percent prescribed) for the 90-day observational period was found to differentiate between various levels of treatment response recorded at study entry (Table 7). On average, patients with suboptimal response had significantly higher mean percentages of imatinib not taken $(23.2 \% \pm 23.8)$ than did those with optimal response $(7.3 \% \pm 19.3, \mathrm{P}=0.005$; percentages calculated as proportions $x 100$ ). Among patients treated with imatinib $\geq 12$ months, those with complete cytogenetic response had significantly lower mean percentages of imatinib not taken $(M=9.0 \% \pm 18.6)$ than those with incomplete cytogenetic response $(\mathrm{M}=26.0 \% \pm 24.4, \mathrm{P}=0.012)$, a treatment result also observed in all patients regardless of length of treatment $(\mathrm{M}=9.1 \% \pm 18.1$ vs. $\mathrm{M}=23.9 \% \pm 19.2, \mathrm{P}=0.004)$. No significant differences in mean percentages of pill count were observed for complete vs. incomplete hematologic response, major vs. less than major molecular response - in all patients and in those treated for 18 or more months. There were no significant differences between response groups in the subjective measures of nonadherence (physician and patient VAS ratings) and adherence with scheduled appointments.

Discussion

While general perceptions of patient adherence to imatinib were uniformly very high among patients, physicians, and third persons, and most patients kept their clinic appointments as scheduled, other measures showed a significant pattern of imatinib nonadherence among CML patients. Using the 4-question BAAS, a clinically useful tool for rapid assessment of potential nonadherence, about one-third of patients qualified as 
nonadherent in the thirty days prior to study enrollment and over the 90-day study period. The percentage of prescribed imatinib taken averaged 90.9 percent with $71.0 \%$ of patients taking less (down to 29\%) but also $14.8 \%$ taking more than prescribed (up to $202 \%$ ). Only $14.2 \%$ were perfectly adherent with $100 \%$ of prescribed imatinib taken.

Based on self-report and pill count, this sample exhibited higher nonadherence than reported in a meta-analysis of 569 studies across 17 diseases (24.8\%), including 65 studies in cancer $(79.1 \%) .{ }^{32}$ This is surprising because of the severity of CML as a disease, the high efficacy of imatinib, its tolerability profile relative to other antineoplastic agents, the high morbidity and mortality of CML prior to the advent of imatinib, and the convenience of oral administration. These factors should be convincing reasons for CML patients to be highly adherent. In general, adherence reduces the risk for null or poor treatment outcomes by $26 \%$, and adherent patients are three times as probable to have good treatment outcomes compared to nonadherent patients. ${ }^{33}$

Apart from a few low but statistically significant correlation coefficients, there was virtually no systematic relationship between adherence to imatinib and time since CML diagnosis, length of imatinib treatment, adverse events (general and suspected to be linked to imatinib), and patient-reported symptoms and their bothersomeness. These findings may run counter to evidence in several other therapeutic areas $17,23,32,33$, however severity of disease, criticality of treatment, and clinical consequences of nonadherence should be taken into account. In this regard, CML patients may be comparable to transplant patients in that there is limited forgiveness of nonadherent behavior and (slight) deviations from the prescribed regimen are associated with poorer clinical outcomes. Where weak significant correlations where observed at follow-up, higher frequencies of general adverse events or greater number of patient-reported symptoms led especially patients to perceive themselves as more adherent - when in fact their actual nonadherence behavior declined. This suggests that assessment of patients' adherence behavior is more important than their 
(already elevated) self-perceptions of adherence. Importantly, how long patients have been diagnosed with CML or how long they have been treated with imatinib was not associated with either perceptions of or actual adherence behavior. This is important because, clinically, one might expect (some) patients to be highly adherent at the beginning of their imatinib treatment but become less so after having been told that they had responded well to treatment. Instead, how adherent a patient is to imatinib may be a stable behavior trait of that patient, rather than one fluctuating with treatment success - though this remains to be studied further.

Nonadherence may be a function of the patient, his/her treating physician, and the center where he/she is being seen. Physician and center accounted for over a third of the variance in patient's self-perceived adherence. This attests to the importance of the patientphysician relationship and patients' positive appraisal of the treatment center. Patients' self-perceived (though inflated) adherence behavior may be an indication in itself of their willingness to actively participate in their care and their self-confidence in doing so. The high adherence with scheduled appointments further underscores this link between patient, physician, and center. The variance explained by physician and center in physicians' subjective perception of patient adherence was much more modest $(6.9 \%$ and $12.0 \%$, respectively); suggesting that for the most part physicians evaluate CML patients' imatinib adherence on a case-by-case basis. Similarly, the modest (9.1\%) amount of variance attributable to treating physicians and the virtually zero percent for center, reinforces the importance of the patient-physician relationship.

Neither perceived adherence rates, self-reported adherence behaviors, nor adherence to scheduled appointments changed significantly over the 90-day study period despite patients' presumably knowing they were participating in an adherence study having given informed consent to such. The high VAS ratings at baseline and follow-up may reflect in part a trend to overestimate adherence by virtue of being enrolled in an adherence study 
or by social desirability bias ${ }^{37}$ to report the presumably correct behavior. However, that rates for self-reported nonadherence behaviors and keeping scheduled appointments did not change over the 90-day period may suggest that imatinib adherence may be a rather stable behavioral trait minimally affected by time or participation in an adherence study.

Congruent with the recommendation for combining adherence assessment methods, ${ }^{23}$ we combined patient self-perceptions, nonadherence behaviors reported by patients, and pill count into a vector of nonadherence. Canonical correlation modeling identified several variables associated with increased nonadherence as well as several variables with a mitigating effect on nonadherence; and this at both the patient and physician levels. Note that these are not independent factors and should be interpreted as part of a canonical model of multiple complementary variables. Demographic variables linked to nonadherence were patients' age, living alone, and being male. Also tied to nonadherence within the context of a multivariate model_were length of time since the diagnosis of CML was made and how long patients had been treated with imatinib (although no univariate statistically significant correlations were observed). This suggests that chronicity of disease and length of treatment may lead some patients to become more lax in their medication behavior. Patients who function relatively well and perceive their chronic care more positively are also more likely to be nonadherent. The association of nonadherence with imatinib dosing of $600 \mathrm{mg} /$ day or higher may be due to the various regimens for this dose: 1 tablet of $400 \mathrm{mg}$ in the morning and 2 capsules of $100 \mathrm{mg}$ in the evening, or 3 capsules of $100 \mathrm{mg}$ in the morning and in the evening. On the other hand, being more knowledgeable about disease and treatment, showing greater self-efficacy in longterm medication behavior, and having at least a secondary school education are all associated with better adherence behavior. Having to take more medications on a daily basis is also associated with imatinib adherence. This is_inconsistent with past findings in other therapeutic areas that the prescribed number of daily doses is inversely related to 
adherence. ${ }^{34}$ Again, severity of disease, criticality of treatment, and seriousness of the clinical consequences of nonadherence may explain our finding. Knowledge and selfefficacy are clinically modifiable factors.

Physicians' experience, practice patterns, and practice environment were found to influence adherence as well. Shorter follow-up visits and years of practicing medicine were associated with increased nonadherence. In contrast, an active CML practice, spending more time with patients at the time of diagnosis, and practicing as a hematologist in a teaching hospital were all related to better adherence.

Doti and colleagues ${ }^{20}$ reported a major cytogenetic response rate $(0-35 \% \mathrm{Ph}-$ positive metaphases) of $89.9 \%$ but did not provide a breakdown in terms of complete versus partial response. Our findings link the percentage of prescribed imatinib not taken to complete vs. partial cytogenetic response and optimal vs. suboptimal treatment response. On average, patients with a lesser response had taken between $74.0 \%$ and $76.8 \%$ of prescribed dose, compared to $89.9 \%$ and $92.7 \%$ for patients with a better response. This constitutes strong initial evidence that nonadherence to imatinib treatment is associated with poorer treatment outcomes, and that $100 \%$ adherence with imatinib treatment is an essential clinical target.

Physicians rated the utility and applicability of thirteen adherence-enhancing strategies higher if these strategies involved active physician participation and/or decisionmaking. Strategies requiring significant patient involvement, whether behaviorally or through the use of assistive devices were perceived as less helpful and applicable in clinical practice. The critical role of patient education was recognized. However, a meta-analysis of adherence-enhancing interventions found that the average effect size of interventions combining educational and behavioral strategies was 0.35 versus 0.20 for educational and 0.22 for behavioral strategies alone. ${ }^{35}$ To improve adherence to imatinib, it will be important to challenge physicians' current beliefs and practices and to develop integrated, 
yet clinically and financially feasible, intervention models. A more pragmatic view on and approach to imatinib nonadherence may also lead to more realistic perceptions of patient adherence and make physicians more aware of the problem.

Adherence behavior, not necessarily perceptions, should be assessed routinely throughout the care of CML patients. Preferably, this should be done as part of the clinical interview and patient questioning should be nonjudgmental and nonaccusatory. If needed, a short interview schedule as the BAAS can serve as a reminder to the clinician of the key questions to be used. If a clinician suspects nonadherence but the patient denies so, additional direct (e.g., assay) and/or indirect methods (e.g., third person report, pill count, prescription refills, electronic monitoring) should be used. ${ }^{23}$ Generally, combined methods have been shown to have greater specificity. ${ }^{36}$

Nonadherence should be examined as a possible reason for patient non- or reduced response to imatinib before considering such patients to be imatinib-resistant and switching them to next-line treatment with the new second-generation tyrosine kinase inhibitors. Nonadherence to imatinib must be ruled out as a possible cause of lack of optimal response.

Our study has several limitations and identifies areas for future research. Of the 202 patients, $169(83.7 \%)$ had evaluable data at the follow-up visit, slightly higher than a recently completed cross-sectional study on adherence to glaucoma treatment conducted in Belgium as well $(80.2 \%) .{ }^{37}$ This may have introduced a selection bias towards patients with better adherence, who can be expected to have more complete data than their nonevaluable counterparts. Patient loss and missing date are not uncommon in observational studies. Relatedly, baseline and follow-up data for cytogenetic and in particular molecular response were censored. The possible bias of good responders to treatment not being tested as frequently as poor responders cannot be excluded. Though adequate for its intent to determine the prevalence of nonadherence, multivariately model determinants, and 
compare the adherence behaviors of patients with better and those with lesser response to treatment, this study's findings must be confirmed and extended in terms of sample size, duration of observation, methods of assessment of nonadherence, and modeling of treatment response as a function of patient-, physician-, and center-level determinants. In the absence of clinically meaningful and empirically validated cut-off to infer nonadherence, all adherence measures except the BAAS were used as continuous variables. Future research should explore meaningful cut-offs for self-reports, pill counts, and VAS ratings for the disease/drug dyad of CML/imatinib. This could be achieved by studying the impact of nonadherence prospectively from both subclinical ${ }^{38}$ and clinical perspectives. This study was limited to one country, for which, in addition, no registry data on the prevalence and incidence of CML are available (informally, the prevalence is estimated at 1000 patients in a total population of 10 million, hence this study captured approximately 1 in 6 CML patients in Belgium). Involving additional countries may have the dual benefit of accruing more patients while better understanding variability in adherence behavior as a function of factors at the healthcare system level. The BAAS may be a helpful tool to assess nonadherence in routine clinical practice. However, in the absence of a validated version for CML, the version used in the ADAGIO study included the two hour deviation of the original immunosuppression version of the scale. This may not be relevant considering the relatively long half-life of imatinib. An appropriately revised version needs to be validated. Future research should complement indirect measures of adherence with direct measures; e.g., imatinib plasma level assays. ${ }^{39}$ The link between nonadherence and cytogenetic response needs to be elucidated further. Our study was limited to 90 days, and it will be necessary to conduct prospective studies to map patterns of medication behavior and their impact on treatment outcomes over longer periods of time, especially for patients with a projected life-long dependence on imatinib. This may also further clarify whether 
adherence to imatinib treatment is a relatively stable behavior trait, or whether there is variability medium and long term over the course of disease and treatment.

To our knowledge, ADAGIO is the first published study of the prevalence, determinants, and clinical outcomes of nonadherence to imatinib treatment in patients with CML. Nonadherence is more prevalent than patients, physicians, and third persons such as spouses and family members believe it is, and is related to poorer rates of response to imatinib. Several determinants may serve as alert signals, while others are clinically modifiable. Patients need to be assessed carefully in terms of their medication behavior and effective yet clinically feasible and cost-effective interventions need to be designed, studied, and implemented.

\section{Acknowledgments}

This study was supported by research funding from Novartis to Matrix45. We thank patients and their families for participating in the study; physician-investigators and their staff for implementing the study; a complete list of participating centers and investigators are listed in the Supplemental Appendix; and Matthew Abraham and Kevin Jones for editorial, proofreading, and manuscript preparation support. We also thank two anonymous reviewers for their thoughtful comments.

Authorship

Contribution

L.N. performed research, enrolled treated patients, collected data, interpreted data, and wrote the manuscript. M.-A.v.L. designed research, performed research, and interpreted 
data, and reviewed the manuscript. R.D.B., G.V., P.Z., Z.B., P. Martiat, P. Mineur, K.V.E., performed research, enrolled treated patients, interpreted data, and reviewed the manuscript. S.D.G. designed research, interpreted data, and wrote the manuscript. I.A. and K.M. designed research, performed research, conducted statistical analysis, interpreted data, and wrote the manuscript. T.A. conducted statistical analysis and wrote the manuscript._. I.A. was the principal investigator. The writing committee included L.N., K.M., S.D.G., and I.A. I.A., K.M., and T.A. vouch for the accuracy and completeness of the data.

Conflict-of-interest disclosure

L. N., R.D.B., G.V., P.Z., P.Martiat, P. Mineur, and K.V.E. report no conflicts of interest. M.-A.vL is an employee of Novartis. I.A., K.M., and T.A. are full- or part-time employees of Matrix45. By company policy, they are prohibited from owning equity in client organizations and performing independent duties for client companies. The research funding was awarded to Matrix45. Matrix45 conducts similar studies for other pharmaceutical companies.

Correspondence

Ivo Abraham, Matrix45, 620 Frays Ridge Road, Earlysville, VA 22936, USA; e-mail: iabraham@matrix45.com. 
References

${ }^{1}$ Hehlmann R, Berger U, Hochhaus A. Chronic myeloid leukemia: a model for oncology. Ann Hematol. 2005;84:487-497.

2 Schindler T, Bornmann W, Pellicena P, et al. Structural mechanism for STI-571 inhibition of abelson tyrosine kinase. Science. 2000;289:1938-1942.

3 Kantarjian H, Sawyers C, Hochhaus A, et al. Hematologic and cytogenetic responses to imatinib mesylate in chronic myelogenous leukemia. N Engl J Med. 2002;346:645-652. [Erratum in: N Engl J Med. 2002;346:1923.]

${ }^{4}$ Druker BJ, Sawyers CL, Kantarjian H, et al. Activity of a specific inhibitor of the BCR-ABL tyrosine kinase in the blast crisis of chronic myeloid leukemia and acute lymphoblastic leukemia with the Philadelphia chromosome. N Engl J Med. 2001;344:1038-1042.

5 Talpaz M, Silver RT, Druker BJ, et al. Imatinib induces durable hematologic and cytogenetic responses in patients with accelerated phase chronic myeloid leukemia: results of a phase 2 study. Blood. 2002;99:1928-1937.

${ }^{6}$ Sawyers CL, Hochhaus A, Feldman E, et al. Imatinib induces hematologic and cytogenetic responses in patients with chronic myeloid leukemia in myeloid blast crisis: results of a phase II study. Blood. 2002;99:3530-3539.

${ }^{7}$ O'Brien S, Guilhot F, Larson R, et al. Imatinib compared with interferon and low-dose cytarabine for newly diagnosed chronic-phase chronic myeloid leukemia. $\mathrm{N}$ Engl J Med. 2003;348:994-1004.

8 O'Brien SG, Guilhot F, Goldman DM et al. International Randomized Study of Interferon Versus STI571 (IRIS) 7-year follow-up: sustained survival, low rate of transformation and increased rate of major molecular response in patients with newly diagnosed chronic myeloid leukemia in chronic phase treated with imatinib. Blood. 2008; 112: Abstract 186.

${ }^{9}$ Baccarani M, Saglio G, Goldman J. Evolving concepts in the management of chronic myeloid leukemia. Recommendations from an expert panel on behalf of The European LeukemiaNet. Blood. 2006;108:1809-1820.

${ }^{10}$ Hahn EA, Glendenning GA, Sorensen MV, et al. Quality of life in patients with newly diagnosed chronic phase chronic myeloid leukemia on imatinib versus interferon alfa plus low-dose cytarabine: results from the IRIS Study. J Clin Oncol. 2003;21:2138-2146.

11 Dalziel K, Round A, Stein K, Garside R, Price A. Effectiveness and cost-effectiveness of imatinib for first-line treatment of chronic myeloid leukaemia in chronic phase: a systematic review and economic analysis. Health Technol Assess. 2004;8:1-120.

12 Curran MP, Croom KF, Goa KL. Imatinib mesylate: a review of its use in chronic myeloid leukemia. American Journal of Cancer. 2003;2:439-454. 
${ }^{13}$ Skrepnek GH, Ballard EE. Cost-efficacy of imatinib versus allogeneic bone marrow transplantation with a matched unrelated donor in the treatment of chronic myelogenous leukemia: a decision-analytic approach. Pharmacotherapy. 2005;25:325-334.

14 Dalziel K, Round A. Garside R. Stein K. Cost effectiveness of imatinib compared with interferon-alpha or hydroxycarbamide for first-line treatment of chronic myeloid leukaemia. Pharmacoeconomics. 2005;23:515-526.

15 Reed SD, Anstrom KJ, Ludmer JA, et al. Cost-effectiveness of imatinib versus interferonalpha plus low-dose cytarabine for patients with newly diagnosed chronic-phase chronic myeloid leukemia. Cancer. 2004;101:2574-2583.

16 Stone RM. Optimizing treatment of chronic myeloid leukemia: a rational approach. Oncologist. 2004;9:259-270.

17 Sabate E. Adherence to long-term therapies: evidence for action. Geneva, World Health Organization. 2003.

18 Halpern R, Barghout V, Mody-Patel N, Williams D. Relationship between compliance, costs, hospitalizations for CML and GIST patients using imatinib mesylate [abstract]. J Clin Oncol. 2008;26:5S. Abstract 6598.

19 Darkow T, Henk HJ, Thomas SK, et al. Treatment interruptions and non-adherence with imatinib and associated healthcare costs: a retrospective analysis among managed care patients with chronic myelogenous leukemia. Pharmacoeconomics. 2007;25:481-496.

20 Doti C, Stemmelin G, Shanley C, et al. Cytogenetic response in relation to the adherence to treatment with imatinib mesylate: a case control study [abstract]. Blood. 2007;110. Abstract 4553.

21 Rudd P. In search of a gold standard for compliance measurement. Arch Intern Med. 1979;139:627-628.

22 DiMatteo MR. Evidence-based strategies to foster adherence and improve patient outcomes. Journal of the American Academy of Physician Assistants. 2004;17:18-21.

23 Osterborg L, Blaschke T. Adherence to medication. New Engl J Med. 2005;353:487-496.

${ }^{24}$ Cleemput I, Dobbels F. Measuring patient-reported outcomes in solid organ transplant recipients: an overview of instruments developed to date. Pharmacoeconomics. $2007 ; 25: 269-286$.

25 De Geest S, Abraham I, Gemoets H, Evers G. Development of the long-term medication behavior self-efficacy scale: qualitative study for item development. J Adv Nurs. 1994;19:233-238.

26 Denhaerynck K, Abraham I, Gourley G, et al. Validity testing of the Long-Term Medication Behavior Self-Efficacy Scale. J Nurs Meas. 2003;11:267-282. 
27 Bandura A. Social Foundations of Thought and Action. Englewood Cliffs, NJ: Prentice Hall;1986.

28 Glasgow RE, Wagner EH, Schaefer J, Mahoney LD, Reid RJ, Greene SM. Development and validation of the Patient Assessment of Chronic Illness Care (PACIC). Med Care. 2005;43:436-444.

${ }^{29}$ Glasgow RE, Whitesides H, Nelson CC, King DK. Use of the Patient Assessment of Chronic Illness Care (PACIC) with diabetic patients: relationship to patient characteristics, receipt of care, and self-management. Diabetes Care. 2005;28:2655-2661.

30 Ware JE, Kosinski M, Dewey JE, Gandek B. How to score and interpret single-item health status measures: a manual for users of the SF-8 Health Survey. Lincoln, RI: QualityMetric Incorporated; 2001.

31 Tabachnick BG ,Fidell LS. Using Multivariate Statistics (4th ed.) Needham Heights, MA: Allyn \& Bacon; 2001.

32 DiMatteo M. Variations in patients' adherence to medical recommendations: a quantitative review of 50 years of research. Med Care. 2004;42:200-209.

33 DiMatteo MR, Giordani PJ, Lepper HS, Croghan TW. Patient adherence and medical treatment outcomes: a meta-analysis. Medical Care. 2002;40:794-811.

${ }^{34}$ Claxton AJ, Cramer J, Pierce C. A systematic review of the associates between dose regimens and medication compliance. Clin Ther. 2001;23:1296-1310.

35 Roter DL, Hall JA, Merisca R, Nordstrom B, Cretin D, Svarstad B. Effectiveness of interventions to improve patient compliance: a meta-analysis. Med Care. 1998;36:11381161.

${ }^{36}$ Schäfer-Keller P, Steiger J, Bock A, Denhaerynck K, De Geest S. Diagnostic accuracy of measurement methods to assess non-adherence to immunosuppressive drugs in kidney transplant recipients. Am J Transplant. 2008;8:616-626.

${ }^{37}$ Vandenbroeck S, Dobbels F, De Geest S, Stalmans I, Zeyen T. Noncompliance, the blind spot in glaucoma treatment: results from the Belgian Compliance Study in Ophthalmology [abstract]. Abstract of oral presentation at the 2007 congress of the Société Belge d'Ophtalmologie - Belgisch Oftalmologisch Gezelschap. http://www.ophthalmologia.be/plugins/abstracts/view_abstract.php?abs_id=1148. Accessed February 24, 2009.

${ }^{38}$ Denhaerynck K, Dobbels F, Cleemput I, et al. Prevalence, consequences, and determinants of nonadherence in adult renal transplant patients: a literature review. Transplant Int. 2005;18:1121-1133.

39 Picard S, Titier K, Etienne G, et al. Trough imatinib plasma levels are associated with both cytogenetic and molecular responses to standard-dose imatinib in chronic myeloid leukemia. Blood. 2007;109:3496-3499. 


\section{Table 1. Evaluation and Visit Schedule}

\begin{tabular}{rrrr}
\hline & visit & 1 & 2 \\
\hline month & 0 & 3 \\
\hline
\end{tabular}

Patient Characteristics

Sociodemographic data

$\mathrm{X}$

Medical history \& current comorbidity

Disease-related Information

Disease history

Current clinical status

$\mathrm{X}$

X X

Concomitant medications

Medications: risk for Rx-Rx interactions

Physician Variables

Sociodemographic data

$\mathrm{X}$

Education

Specialty

Practice environment

Number of active CML patients

Time spent with patients: newly diagnosed (first consultation)

Time spent with patients: during treatment

Use of scientific information / evidence-based practice

Use of patient awareness and support materials

Perspectives on patient compliance

$\mathrm{X}$

$\mathrm{X}$

$\mathrm{X}$

$\mathrm{X}$

$\mathrm{X}$

$\mathrm{X}$

$\mathrm{X}$

$\mathrm{X}$

$\mathrm{X}$

Patient Compliance

Basel Assessment of Adherence Scale, Visual Analog Scale - 30 days retrospective - patient interview

Basel Assessment of Adherence Scale, Visual Analog Scale - 30 days retrospective - family member interview

Pill count (delta grams taken vs. grams prescribed) - 90 days retrospective

Appointment noncompliance - 30 days retrospective

Physician Visual Analog Scale rating of patient compliance - 30 days retrospective

Patient Variables

Long-Term Medication Behavior Self-Efficacy Scale (adapted for CML/imatinib)

Patient Assessment of Chronic Illness Care (PACIC) - 30 days retrospective

Symptom experience and distress -30 days retrospective

Understanding of disease and treatment

Functional status / Quality of Life (SF-8) - 30 days retrospective

Patient knowledge-seeking behavior - 90 days retrospective

$\begin{array}{ll}X & X \\ X & X \\ & X \\ X & X \\ X & X\end{array}$

$\mathrm{X}$

$\mathrm{X} \quad \mathrm{X}$

$\mathrm{X} \quad \mathrm{X}$

$\mathrm{X} \quad \mathrm{X}$

$\mathrm{X} \quad \mathrm{X}$

$\mathrm{X}$

Response Parameters

CML: hematological response, cytogenetic response, molecular response

$\mathrm{X} \quad \mathrm{X}$

Treatment-related

Number of visits to GP between visit 1 and visit 2 related to CML/imatinib

Number of visits to specialist between visit 1 and visit 2 related to CML/imatinib 
Table 2. Patient Demographics and Clinical Status at Baseline

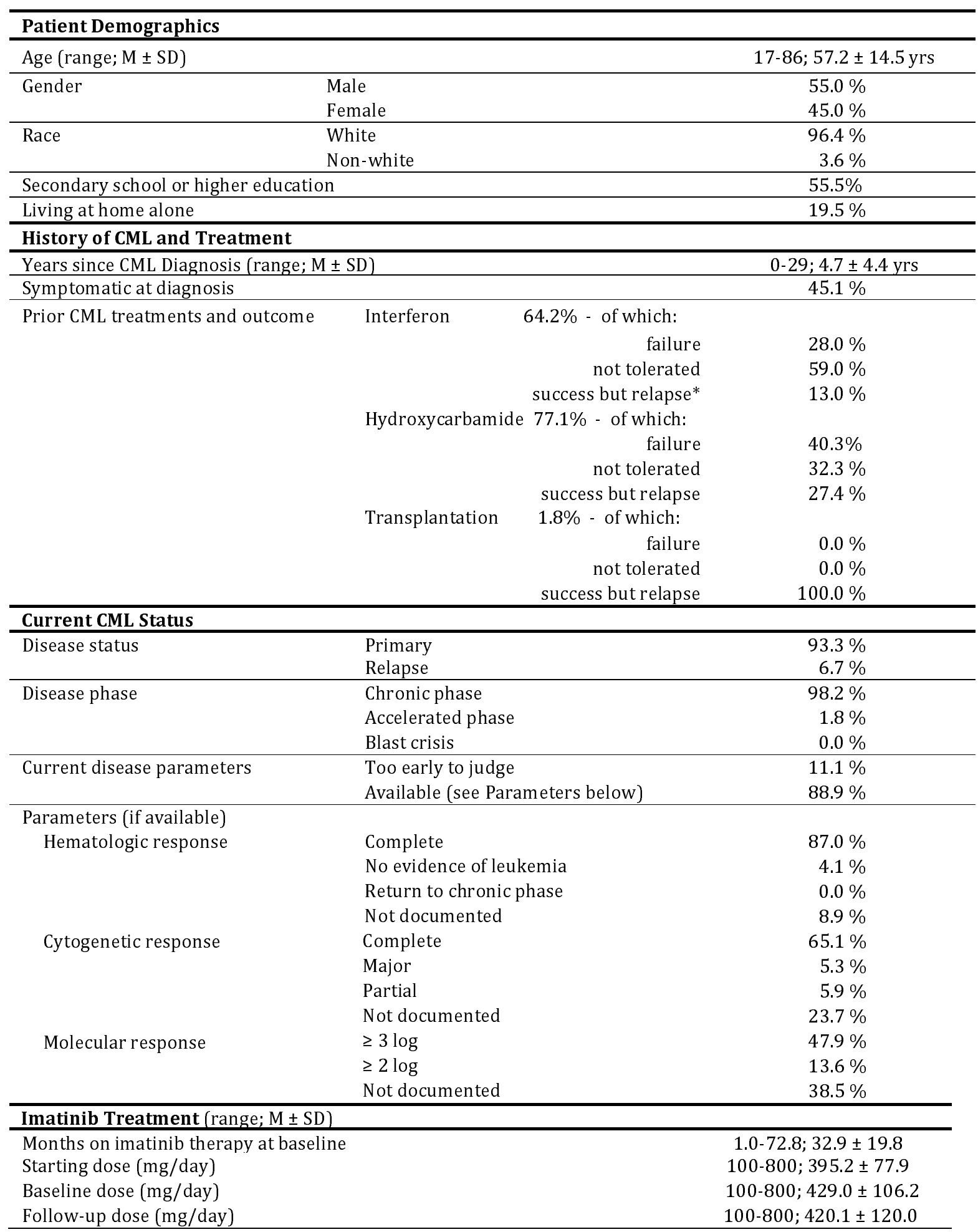




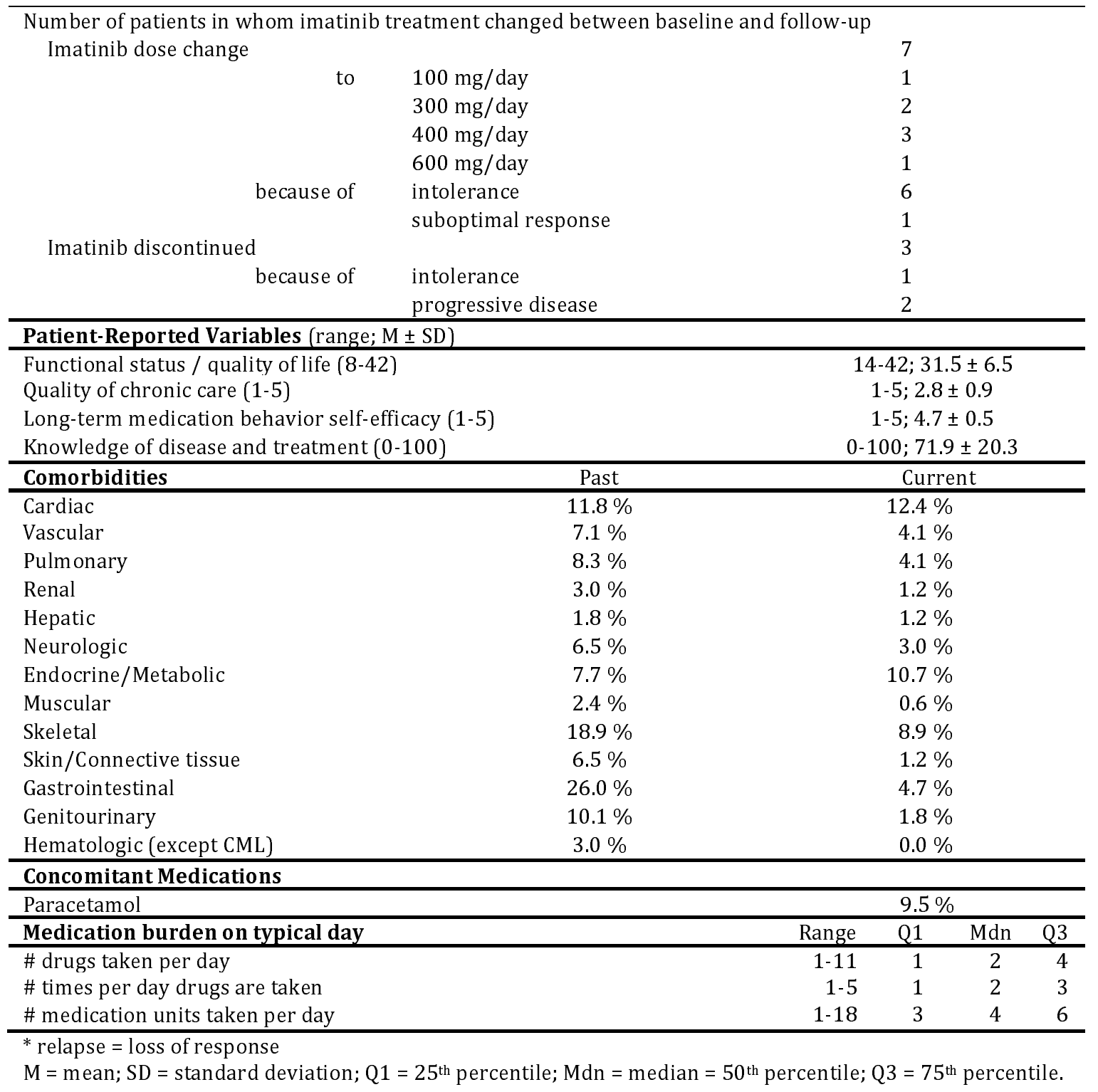


Table 3. Disease Phase, Disease Parameters and Patient-Reported Outcomes at Baseline and Follow-up

\begin{tabular}{|c|c|c|c|c|c|c|c|}
\hline Disease phase & Baseline & & \multicolumn{3}{|c|}{ Follow-up } & & $\mathbf{P}$ \\
\hline Chronic phase & \multicolumn{2}{|l|}{$98.2 \%$} & \multicolumn{3}{|c|}{$99.4 \%$} & \multicolumn{2}{|r|}{ n.s. } \\
\hline Accelerated phase & \multicolumn{2}{|l|}{$1.8 \%$} & \multicolumn{3}{|c|}{$0.6 \%$} & & \\
\hline Blast crisis & \multicolumn{2}{|l|}{$0.0 \%$} & \multicolumn{3}{|c|}{$0.0 \%$} & & \\
\hline \multicolumn{8}{|l|}{ Disease parameters (if available)* } \\
\hline Hematologic response & \multicolumn{2}{|l|}{$\mathrm{n}=154$} & \multicolumn{3}{|c|}{$\mathrm{n}=144$} & \multicolumn{2}{|r|}{ n.s. } \\
\hline Complete & \multicolumn{2}{|l|}{$95.5 \%$} & \multicolumn{3}{|c|}{$94.4 \%$} & & \\
\hline No evidence of leukemia & \multicolumn{2}{|l|}{$4.5 \%$} & \multicolumn{3}{|c|}{$2.8 \%$} & & \\
\hline Return to chronic phase & \multicolumn{2}{|l|}{$0.0 \%$} & \multicolumn{3}{|c|}{$2.8 \%$} & & \\
\hline Cytogenetic response & \multicolumn{2}{|l|}{$\mathrm{n}=129$} & \multicolumn{3}{|c|}{$\mathrm{n}=\mathbf{1 1 0}$} & \multicolumn{2}{|r|}{ n.s. } \\
\hline Complete & \multicolumn{2}{|l|}{$85.3 \%$} & \multicolumn{3}{|c|}{$87.3 \%$} & & \\
\hline Major & \multicolumn{2}{|l|}{$7.0 \%$} & \multicolumn{3}{|c|}{$6.4 \%$} & & \\
\hline Partial & \multicolumn{2}{|l|}{$7.8 \%$} & \multicolumn{3}{|c|}{$6.4 \%$} & & \\
\hline Molecular response & \multicolumn{2}{|l|}{$n=104$} & \multicolumn{3}{|c|}{$n=96$} & \multicolumn{2}{|r|}{ n.s. } \\
\hline$\geq 3 \log$ & \multicolumn{2}{|l|}{$77.9 \%$} & \multicolumn{3}{|c|}{$75.0 \%$} & & \\
\hline \multirow[t]{3}{*}{$\geq 2 \log$} & \multicolumn{2}{|l|}{$22.1 \%$} & \multicolumn{3}{|c|}{$25.0 \%$} & & \\
\hline & & seline & & & low-ul & & $\mathbf{P}$ \\
\hline & Range & $\mathbf{M}$ & SD & Range & $\mathbf{M}$ & SD & \\
\hline Functional status / quality of life $(8-42)^{* *}$ & $14-42$ & 31.5 & 6.5 & $16-42$ & 32.3 & 6.5 & n.s. \\
\hline Quality of chronic care (1-5) & $1-5$ & 2.8 & 0.9 & $1-4.9$ & 2.6 & 0.9 & 0.01 \\
\hline Knowledge $(0-100)$ & $0-100$ & 71.9 & 20.3 & $0-100$ & 69.2 & 24.4 & n.s. \\
\hline
\end{tabular}


Table 4. Physicians' Perceptions of Utility and Applicability of Adherence-Enhancing Strategies

\begin{tabular}{|c|c|c|c|c|}
\hline \multirow{3}{*}{$\begin{array}{l}\text { Adherence-Enhancing } \\
\text { Strategies }\end{array}$} & \multicolumn{3}{|c|}{ Utility (0-3) } & \multirow{2}{*}{$\begin{array}{c}\text { Applicability } \\
\text { in Practice (0-5) }\end{array}$} \\
\hline & Effectiveness & Feasibility & Cost & \\
\hline & $\mathrm{M} \pm \mathrm{SD}$ & $\mathrm{M} \pm \mathrm{SD}$ & $\mathrm{M} \pm \mathrm{SD}$ & $\mathrm{M} \pm \mathrm{SD}$ \\
\hline $\begin{array}{l}\text { Treatment selection based on } \\
\text { patient characteristics }\end{array}$ & $1.9 \pm 1.1$ & $1.6 \pm 1.1$ & $1.3 \pm 1.0$ & $1.0 \pm 1.5$ \\
\hline Patient education & $2.6 \pm 0.6$ & $2.1 \pm 0.8$ & $1.4 \pm 0.8$ & $3.1 \pm 1.9$ \\
\hline $\begin{array}{l}\text { Improved patient-physician } \\
\text { communication }\end{array}$ & $2.7 \pm 0.5$ & $2.3 \pm 0.8$ & $1.3 \pm 0.8$ & $2.8 \pm 1.7$ \\
\hline $\begin{array}{l}\text { Simplifying medication } \\
\text { regimen }\end{array}$ & $2.5 \pm 0.7$ & $2.3 \pm 0.7$ & $1.6 \pm 0.9$ & $2.3 \pm 1.9$ \\
\hline $\begin{array}{l}\text { Self-monitoring of health } \\
\text { status }\end{array}$ & $1.7 \pm 0.8$ & $1.5 \pm 0.8$ & $1.1 \pm 0.9$ & $0.4 \pm 1.1$ \\
\hline $\begin{array}{l}\text { Maintaining a health status } \\
\text { diary }\end{array}$ & $1.7 \pm 0.8$ & $1.5 \pm 0.8$ & $1.0 \pm 0.8$ & $0.5 \pm 1.3$ \\
\hline Memory cues & $1.7 \pm 0.8$ & $1.9 \pm 0.8$ & $1.0 \pm 0.8$ & $0.6 \pm 1.3$ \\
\hline Spouse / family involvement & $2.2 \pm 0.9$ & $2.0 \pm 0.9$ & $0.6 \pm 0.8$ & $1.3 \pm 1.4$ \\
\hline Regular physician contact & $2.4 \pm 0.5$ & $2.2 \pm 0.7$ & $1.7 \pm 0.8$ & $2.2 \pm 1.8$ \\
\hline $\begin{array}{l}\text { Monitoring of patient } \\
\text { compliance by physician }\end{array}$ & $2.2 \pm 0.7$ & $1.8 \pm 0.9$ & $1.4 \pm 0.8$ & $0.7 \pm 1.1$ \\
\hline Electronic reminder system & $1.5 \pm 0.9$ & $1.2 \pm 0.8$ & $2.3 \pm 0.9$ & $0.3 \pm 1.1$ \\
\hline $\begin{array}{l}\text { Electronic medication } \\
\text { monitors }\end{array}$ & $1.6 \pm 0.9$ & $1.3 \pm 0.7$ & $2.3 \pm 0.9$ & $0.3 \pm 1.1$ \\
\hline $\begin{array}{l}\text { Providing rewards for good } \\
\text { adherence }\end{array}$ & $1.4 \pm 1.0$ & $1.3 \pm 0.8$ & $1.8 \pm 0.9$ & $0.5 \pm 1.4$ \\
\hline
\end{tabular}

$\mathrm{M}=$ mean; $\mathrm{SD}=$ standard deviation 
Table 5. Adherence at Baseline and Follow-up

\begin{tabular}{|c|c|c|c|c|c|c|c|c|}
\hline \multirow{2}{*}{ Visual Analogue Scale } & & \multicolumn{3}{|c|}{ Baseline } & \multicolumn{3}{|c|}{ Follow-up } & \multirow[t]{2}{*}{ Pacross } \\
\hline & & Range & $M$ & SD & Range & $\mathrm{M}$ & SD & \\
\hline Physician ( $\mathrm{N}=167)$ & & $60-100$ & 94.9 & 7.7 & $0-100$ & 94.9 & 9.9 & n.s. \\
\hline Patient ( $\mathrm{N}=169)$ & & $25-100$ & 95.3 & 8.5 & $75-100$ & 95.7 & 6.1 & n.s. \\
\hline \multirow[t]{2}{*}{ Family (N=79) } & & $80-100$ & 97.0 & 5.0 & $75-100$ & 97.1 & 5.4 & n.s. \\
\hline & $P_{\text {down }}$ & \multicolumn{3}{|c|}{ n.s. } & \multicolumn{3}{|c|}{ n.s. } & \\
\hline
\end{tabular}

Basel Assessment of Adherence Scale

$\%$ of patients $(\mathrm{N}=165)$ who in past 4 weeks...

\begin{tabular}{lccc}
\hline Dose not taken & $16.1 \%$ & $13.3 \%$ & n.s. \\
Consecutive doses not taken & $3.0 \%$ & $1.8 \%$ & n.s. \\
Dose taken with $>$ hour delay & $22.2 \%$ & $25.3 \%$ & n.s. \\
Dose reduced & $1.2 \%$ & $1.8 \%$ & n.s. \\
\hline$\%$ of nonadherent patients & $36.1 \%$ & $32.7 \%$ & n.s. \\
$\%$ of adherent patients & $63.9 \%$ & $67.3 \%$ & n.s. \\
\hline
\end{tabular}

\begin{tabular}{|c|c|c|c|c|c|}
\hline \multicolumn{6}{|c|}{ Adherence with scheduled appointments $(\mathrm{N}=82)^{1}$} \\
\hline$\%$ of adherent patients & $89.4 \%$ & \multicolumn{3}{|c|}{$86.6 \%$} & n.s. \\
\hline$\%$ of appointments kept (range, $\mathrm{M} \pm \mathrm{SD}$ ) & $0-100 ; 90.9 \pm 28.6$ & \multicolumn{3}{|c|}{$0-150 ; 92.1 \pm 28.8$} & n.s. \\
\hline \multicolumn{2}{|l|}{ Pill Count at follow-up $(\mathrm{N}=162)$} & Range & M & SD & \\
\hline \multicolumn{2}{|c|}{$\%$ of prescribed dose taken from baseline to follow-up } & $29-202$ & 90.9 & 20.1 & \\
\hline
\end{tabular}

\footnotetext{
${ }^{1}$ Applies only to patients with scheduled appointments in 30 days prior to baseline and/or follow-up $\mathrm{M}=$ mean; $\mathrm{SD}$ = standard deviation; $\mathrm{P}_{\text {across }}=$ probability of test statistic for test comparing baseline and follow-up; $\mathrm{P}_{\text {down }}=$ probability of test statistic for test comparing within time point; $\mathrm{N}=$ sample size (available observations)
} 


\section{Table 6. Canonical Correlation Analysis of Set of Nonadherence Measures with Set of Determinants}

\begin{tabular}{|c|c|}
\hline $\begin{array}{l}\text { Determinants of } \\
\text { Increased Nonadherence }\end{array}$ & $\begin{array}{c}\text { Canonical } \\
\text { Loading }\end{array}$ \\
\hline \multicolumn{2}{|l|}{ Patient-related } \\
\hline Age & 0.649 \\
\hline Months since diagnosis of CML & 0.272 \\
\hline Living alone & 0.246 \\
\hline Male gender & 0.194 \\
\hline Months on imatinib & 0.193 \\
\hline Imatinib dose $\geq 600 \mathrm{mg} /$ daily & 0.193 \\
\hline Quality of chronic care & 0.125 \\
\hline Functional status / quality of life* & 0.117 \\
\hline \multicolumn{2}{|l|}{ Physician-related } \\
\hline Median duration of treatment follow-up visits & 0.237 \\
\hline Years of professional experience & 0.135 \\
\hline $\begin{array}{l}\text { Determinants of } \\
\text { Decreased Nonadherence }\end{array}$ & $\begin{array}{c}\text { Canonical } \\
\text { Loading }\end{array}$ \\
\hline \multicolumn{2}{|l|}{$\underline{\text { Patient-related }}$} \\
\hline Knowledge of disease and treatment & -0.314 \\
\hline Number of medications taken per day & -0.184 \\
\hline Secondary school graduate & -0.140 \\
\hline Long-term medication behavior self-efficacy & -0.062 \\
\hline \multicolumn{2}{|l|}{ Physician-related } \\
\hline Number of CML patients seen in past year & -0.363 \\
\hline Median duration of first visit with newly diagnosed CML patient & -0.119 \\
\hline Practicing in university or university-affiliated hospital & -0.003 \\
\hline Hematologist & -0.002 \\
\hline \multicolumn{2}{|l|}{ Canonical Model Parameters } \\
\hline $\begin{array}{l}\text { Canonical correlation } \\
\quad \text { Wilk's test }=0.484, \chi^{2}=74.04, P=0.036\end{array}$ & 0.509 \\
\hline Redundancy proportion & 0.015 \\
\hline
\end{tabular}


From www.bloodjournal.org at BIOMEDISCHE BIBLIOTHEEK on May 10, 2009. For personal use only.

Table 7. Nonadherence (Pill Count) and Treatment Response

\begin{tabular}{lcccc}
\hline & $\mathrm{n}$ & $\mathrm{M}$ & $\mathrm{SD}$ & $\mathrm{P}$ \\
\hline All Patients & & & & 0.005 \\
$\quad$ Optimal Response & 124 & $7.3 \%$ & $19.3 \%$ & \\
$\quad$ Suboptimal Response & 14 & $23.2 \%$ & $23.8 \%$ & \\
\hline Patients treated with Imatinib $\geq 12$ months & & & & 0.012 \\
$\quad$ Complete Cytogenetic Response & 98 & $9.0 \%$ & $18.6 \%$ & \\
$\quad$ Incomplete Cytogenetic Response & 9 & $26.0 \%$ & $24.4 \%$ & \\
\hline All Patients & 109 & $9.1 \%$ & $18.1 \%$ & \\
$\quad$ Complete Cytogenetic Response & 15 & $23.9 \%$ & $19.2 \%$ & \\
$\quad$ Incomplete Cytogenetic Response & & &
\end{tabular}

